\title{
Proteomics analysis for the identification of three species of Thunnus
}

\author{
T. Pepe • M. Ceruso $\cdot$ A. Carpentieri $\cdot$ I. Ventrone $\bullet$
}

A. Amoresano • A. Anastasio

Published online: 18 May 2010

(C) Springer Science+Business Media B.V. 2010

\begin{abstract}
The genus Thunnus comprises many species, some of higher quality and commercial value for their excellent organoleptic features, while others of lower quality and value. Consequently, these species are subjected to frequent fraudulent substitution. Increasing trade in fillet and minced fish makes the identification of external anatomical and morphological features of fish impossible. Proteomics was used for the identification of three Thunnus species. Muscle extracts were evaluated by both mono- and two-dimensional electrophoresis and mass spectrometric techniques. Preliminary results demonstrate that the tested species displays a high degree of polymorphism, making possible an accurate identification.
\end{abstract}

Keywords Proteomics species identification Thunnus genus ·

Two-dimensional electrophoresis

\author{
Abbreviations \\ 2DE \\ IEF \\ two-dimensional electrophoresis \\ PCR polymerase chain reaction \\ MALDI-TOF Matrix-assisted laser desorption/ionization-time of flight \\ SDS-PAGE sodium dodecylsufate-polyacrylamide gel electrophoresis
}

T. Pepe $(\bowtie) \cdot$ M. Ceruso $\cdot$ I. Ventrone $\cdot$ A. Anastasio

Dipartimento di Scienze Zootecniche e Ispezione degli Alimenti, Sezione di Ispezione, Napoli, Italy

e-mail: tiziana.pepe@unina.it

A. Carpentieri · A. Amoresano

Dipartimento di Chimica organica e Biochimica Università degli Studi di Napoli "Federico II", Napoli, Italy 


\section{Introduction}

Species belonging to the Thunnus genus often have different qualities and commercial values. The increasing trade of tuna fillets makes fish species identification difficult because their morphological characteristics are partially or completely lost during processing. Several analytical techniques may be used to study and identify prepared fish products. Among these methods, capillary zone electrophoresis (Acuña et al. 2008), isoelectric focusing (IEF) of sarcoplasmic proteins (Rehbein et al. 2000), and amplification of specific DNA fragments, using polymerase chain reaction (PCR) appear to be the most suitable techniques (Pepe et al. 2007).

However, these methods are unable to identify closely related species (e.g., Thunnus). Proteomics analysis has been applied to fish products to examine water-soluble muscle proteins from farm and wild fish to show aquaculture effects on seafood quality (Monti et al. 2005). Only recently, proteomics was considered for species identification in seafood products (Carrera et al. 2007).

A classical proteomics approach based on the analysis of muscle proteins by two-dimensional electrophoresis (2DE) allows more accurate fish protein characterization than IEF. Such analyses characterize sarcoplasmic proteins with both their isoelectric points and molecular weights (Piñeiro et al. 1999). In this study, we applied proteomics analyses, for the first time, to the identification of three tuna species (i.e., T. thynnus, T. alalunga, and T. albacares). Sarcoplasmic proteins were studied by 1- and 2DE, and the most significant proteins for the characterization of the three species were analyzed subsequently by mass spectrometry.

\section{Materials and methods}

In this study, six tuna specimens were analyzed, two $T$. thynnus fished in the Mediterranean Sea and supplied by the "Pozzuoli fish market," two T. albacares from Ecuador, and two T. alalunga from Palermo. Total sarcoplasmic proteins were extracted from fish muscle ( $3 \mathrm{~g}$ ) according to the method of Carrera et al. (2007). Total protein content was measured by the BioRad method. From each tuna species, two different protein aliquots $(50$ and $10 \mu \mathrm{g}$ ) were separated by sodium dodecylsufate-polyacrylamide gel electrophoresis (SDS-PAGE). IEF was performed using 7-cm gel strips in the nonlinear $\mathrm{pH}$ 3-10 range. In the second dimension (SDS-PAGE), the proteins were separated on a $12.5 \%(\mathrm{w} / \mathrm{w})$ polyacrylamide gel, at a constant current of $25 \mathrm{~mA}$. Gels were stained with Coomassie Brilliant Blue R-250 dye. ImageMaster ${ }^{\mathrm{TM}}$ 2D Platinum software was used for the analysis of the 2D gel images.

The most significant spots for species identification, revealed by the software, were excised from the SDS gel and subjected to an in situ protein hydrolysis protocol. Matrixassisted laser desorption/ionization-time of flight (MALDI-TOF) mass spectra of the peptide mixtures were recorded using a Voyager DE-STR MALDI-TOF instrument (Applied Biosystem) operating in reflector mode. The mass spectra obtained were used for protein identification using the Mascot software. The software compares peptide masses obtained by MALDI-TOF for each spot with the theoretical peptide masses from all proteins included in the peptide mass fingerprinting databases. The National Center for Biotechnology Information (NCBI) databank was accessed, restricting analysis to the pertinent taxonomies. The scarce number of tuna sequences deposited in the database limited the identification of proteins. Therefore, the obtained protein sequences were analyzed for homologies with other fish species in the NCBI database using BLAST. 


\section{Results}

SDS-PAGE allowed an evaluation of the efficacy of the extraction protocol and revealed certain differences among the protein patterns of the analyzed tuna species. Protein extracts showed qualitative, inter-species differences, in particular for protein bands with molecular weights less than $10 \mathrm{kDa}$.

The results obtained by 2DE demonstrate noticeable inter-species differences from the assignment and distribution of the electrophoretic spots, even after visual observation. The analysis of the 2D gel revealed the presence of a protein with a molecular weight of approximately $70 \mathrm{kDa}$ in the T. thynnus $2 \mathrm{DE}$ pattern, which was absent in the other species. This protein, identified as Trioso fosfato isomerasi (gi46909469) by mass spectrometry might be considered a specific marker. Other proteins in the T. albacares and T. alalunga 2DE patterns were revealed as species-specific, and similar analyses are currently underway.

\section{Discussion}

Proteomics appears to be a useful method to increase our knowledge on proteins from foodproducing animals (Pandey and Mann 2000). The preliminary results from both 1- and 2DE show significant inter-species differences. In fact, proteomic fingerprinting of three species from the Thunnus genus was obtained. For T. Thynnus, analysis revealed the presence of species-specific proteins that might be used as specific markers. This study offers a new tool for species identification of raw and minced fish. From species-specific proteins, it might be possible to identify species-specific genes and design primers for routine molecular biology methods for processed fish products.

\section{References}

Acuña G, Ortiz-Riaño E, Vinagre J, García L, Kettlun AM, Puente J, Collados L, Valenzuela MA (2008) Application of capillary electrophoresis for the identification of Atlantic salmon and rainbow trout under raw and heat treatment. J Capill Electrophor Microchip Technol 10:93-99

Carrera M, Cañas B, Piñeiro C, Vázquez J, Gallardo JM (2007) De novo mass spectrometry sequencing and characterization of species-specific peptides from nucleoside diphosphate kinase B for the classification of commercial fish species belonging to the family Merlucciidae. J Proteome Res 6:3070-3080

Monti G, De Napoli L, Mainolfi P, Barone R, Guida M, Marino G, Amoresano A (2005) Monitoring food quality by microfluidic electrophoresis, gas chromatography, and mass spectrometry techniques: Effects of aquaculture on the sea bass (Dicentrarchus labrax). Anal Chem 77:2587-2594

Pandey A, Mann M (2000) Proteomics to study genes and genomes. Nature 405:837-846

Pepe T, Trotta M, Di Marco I, Anastasio A, Bautista JM, Cortesi ML (2007) Fish species identification in surimi-based products. J Agric Food Chem 55:3681-3685

Piñeiro C, Barros-Velázquez J, Stelo CG, Gallardo JM (1999) The use of two-dimensional electrophoresis in the characterization of the water-soluble protein fraction of commercial flat fish species. Z Lebensm Unters Forsch 208:342-348

Rehbein H, Kündiger R, Pineiro C, Perez-Martin RI 2000. Fish muscle parvalbumins as marker proteins for native and urea isoelectric focusing. Electrophoresis 21:1458-1463 